\title{
Acute Appendicitis Due to Metastasis of Prostatic Adenocarcinoma: A Case Report
}

\author{
Imad El Moussaoui ${ }^{\mathrm{a}, \mathrm{b}}$, Romain Diamand ${ }^{\mathrm{a}}$, Manke Dika ${ }^{\mathrm{a}}$, Augustin Limbga ${ }^{\mathrm{a}}$, \\ Abdelilah Mehdi ${ }^{\mathrm{a}}$
}

\begin{abstract}
Acute appendicitis due to metastasis of prostatic carcinoma is very rare, and only five cases of metastasis of prostatic carcinoma are described in the literature. We report the case of a 73-year-old man with a history of an adenocarcinoma of the prostate with multiple bone metastases. The patient was admitted to the emergency department with symptoms and signs of an acute appendicitis which was confirmed by computed tomography (CT). Laparoscopic surgical exploration was performed, affirming an acute appendicitis with a suspicious lesion in the appendix base. Due to the location of the lesion, an ileocecectomy was performed. Histopathological and immunohistochemical examinations of the specimen showed an extrinsic infiltration of the appendix by adenocarcinoma metastasis expressing the prostate-specific antigen (PSA). These results confirmed an acute appendicitis caused by metastasis of prostatic adenocarcinoma.
\end{abstract}

Keywords: Metastasis; Prostate; Appendix

\section{Introduction}

Acute appendicitis is one of the most common reasons for surgical abdominal interventions. Obstruction of the appendiceal lumen with fecaliths is the most common cause of appendicitis. Tumors, benign and malignant of the appendix are uncommon. Metastatic tumors inducing appendicitis are extremely rare.

To the best of our knowledge, only five other cases of appendicular metastases caused by a prostatic carcinoma have been reported, and our case is the first to visualize prostatic metastasis in an acute appendicitis and the unique case treated with ileocecectomy.

Manuscript accepted for publication October 08, 2015

aDepartment of General Surgery, Free University of Brussels, Etterbeek-Ixelles Hospital, 1050 Brussels, Belgium

${ }^{\mathrm{b} C}$ Corresponding Author: Imad El Moussaoui, Department of Surgery, Etterbeek-Ixelles Hospital, Rue Jean Paquot 63, 1050 Brussels, Belguim.

Email: imadimad999@hotmail.com

doi: http://dx.doi.org/10.14740/jcs284w

\section{Case Report}

A 73- year-old man was admitted to the emergency department with right iliac fossa pain for 5 days without nausea, vomiting or fever. His medical history indicated a prostatic adenocarcinoma with multiple bone metastases located in spine, ribs and sternum. Six years ago, the patient underwent an ultrasoundguided needle biopsy after a suspicious digital rectal examination, which yielded a poorly differentiated prostate adenocarcinoma with Gleason score of 7 . A radical prostatectomy was performed with a finding of positive regional lymph nodes, associated to doxorubicin, cyclophosphamide chemotherapy, and gonadotropin-releasing hormone therapy administration.

Physical examination revealed rebound tenderness over McBurney's point. Laboratory tests found leukopenia with a white blood count of 3,700/ $\mu \mathrm{L}$ (normal range: 4,000 - 10,000/ $\mu \mathrm{L}$ ), an elevated C-reactive protein level to $45.5 \mathrm{mg} / \mathrm{L}$ (normal range: $<6 \mathrm{mg} / \mathrm{L}$ ), and no alterations in liver tests and no other significant biological abnormalities were observed. Vital signs were normal. Clinical examination showed pain within deep palpation of the right iliac fossa with rebound tenderness, and the rest of the examination was unremarkable. Contrastenhanced computed tomography (CT) revealed a dilated appendix with a size of $11 \mathrm{~mm}$ in diameter, and a diffuse infiltration of surrounding fat (Fig. 1), and multiple bone metastases are also visible.

Consequently, an urgent laparoscopic surgical exploration was performed; this exploration featured an acute appendicitis with a suspicious lesion at the appendicular base. In this case because of the suspicious lesion's location, a simple cecectomy was impossible due to the risk of stenosis at the last ileal loop. An ileocecectomy with side-to-side stapled ileo-colonic anastomosis was performed after conversion to laparotomy.

The postoperative course was slow but favorable with oral feeding started on the first postoperative day. On the sixth postoperative day, the patient developed a postoperative ileus which required nasogastric decompression and parenteral nutrition for 7 days until resolution of the ileus and the resumption of enteral nutrition.

Histopathological examination showed an extrinsic infiltration of the appendix by an adenocarcinoma metastasis with surgical free margins. Immunohistochemical staining revealed that the tumor cells expressed the prostate-specific antigen (PSA) strongly. 


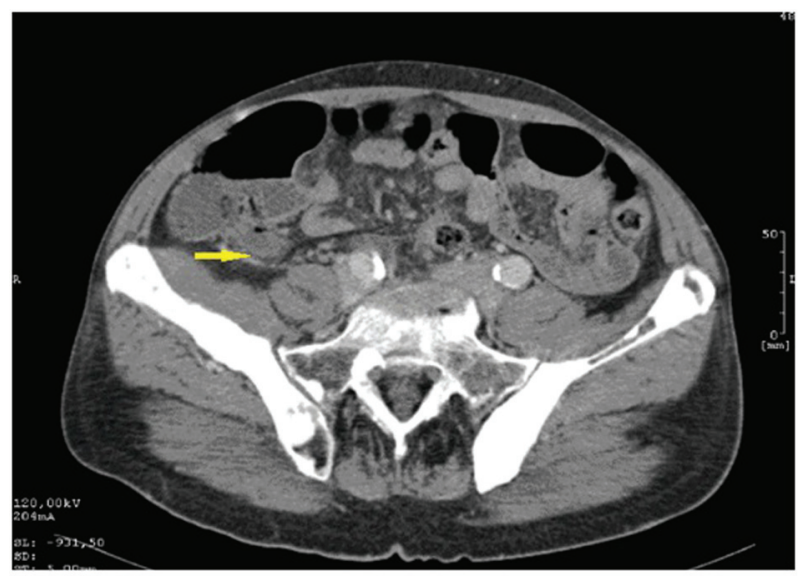

Figure 1. Abdominal computed tomography showing distended appendix, measuring $11 \mathrm{~mm}$ in diameter and diffuse infiltration of surrounding fat.

These results confirmed the assumption of prostatic metastases which were suggested because of the patient's medical history.

The patient was transferred to the oncology department for further treatment.

\section{Discussion}

Acute appendicitis is one of the most common causes of acute abdomen and one of the most common indications for surgical abdomen. It is mainly caused by an obstruction of appendiceal lumen which leads to an increase in the intraluminal pressure and luminal distention, causing a venous engorgement, arterial compromise, tissue ischemia, and bacterial growth. Continuation of this process results in appendiceal infarction as well as perforation of the appendix [1].

The obstruction is usually caused by fecaliths, and other causes are described as lymphoid hyperplasia, foreign bodies, endometriosis and parasites.

The primary tumors of the appendix are rare, occurring in only $1 \%$ of appendicectomies [2]. The acute appendicitis derived from metastases is uncommon and exceptional, as it is an incidental finding in only $0.01 \%$ of appendicectomies [3]. In recent literature review, only seven patients were found to have metastatic appendiceal cancer among 80,698 patients [4].

Metastasis to the appendix usually originates in carcinomas of the gastrointestinal tract, breast, lung or female genital tract. Only five cases of appendicular metastasis of prostate cancer are described in the literature [5-9].

The mechanism of acute appendicitis is caused by an obstruction of the lumen of the appendix by metastatic tumor. The perforation rate of an acute appendicitis within patients with metastases is $71 \%$, whereas simple appendicitis shows a perforation rate which varies between $17 \%$ and $39 \%$ [10]. This high rate of perforation may be explained by the effect of immunosuppression in cancer patients and the local effect of the tumor which decreases the capacity of the appendix to limit inflammation and obstruction of the lumen.
The diagnosis of an acute appendicitis derived from metastasic prostate cancer is a histopathological diagnosis using immunohistochemical staining showing tumor cells expressing PSA.

In other case reports [5-9], the appendix did not show any visible suspicious lesions and therefore only an appendectomy was performed. In spite of this, our case report is the first to visualize the metastasis during the operation and perform an ileocecal resection on acute appendicitis to metastatic carcinoma of the prostate.

With the improvement in survival of cancer patients, we will face an increase in acute appendicitis caused by metastasis.

\section{Conclusion}

Although the tumor etiology in acute appendicitis is rare, it should be included in the differential diagnosis if an oncological patient presents symptoms and signs of an acute appendicitis.

\section{Conflict of Interests}

The authors declared that there is no conflict of interests regarding this study.

\section{References}

1. Birnbaum BA, Wilson SR. Appendicitis at the millennium. Radiology. 2000;215(2):337-348.

2. McCusker ME, Cote TR, Clegg LX, Sobin LH. Primary malignant neoplasms of the appendix: a population-based study from the surveillance, epidemiology and end-results program, 1973-1998. Cancer. 2002;94(12):3307-3312.

3. Connor SJ, Hanna GB, Frizelle FA. Appendiceal tumors: retrospective clinicopathologic analysis of appendiceal tumors from 7,970 appendectomies. Dis Colon Rectum. 1998;41(1):75-80.

4. Akbulut S, Tas M, Sogutcu N, Arikanoglu Z, Basbug M, Ulku A, Semur H, et al. Unusual histopathological findings in appendectomy specimens: a retrospective analysis and literature review. World J Gastroenterol. 2011;17(15):1961-1970.

5. Stein A, Sova Y, Almalah I, Lurie A. The appendix as a metastatic target for male urogenital tumours. Br J Urol. 1996;78(4):647-648.

6. Ratanarapee S, Nualyong C. Acute appendicitis as primary symptom of prostatic adenocarcinoma: report of a case. J Med Assoc Thai. 2010;93(11):1327-1331.

7. Grau J, Rinker B, Sidhu G, Kim A, Newman E. Acute appendicitis secondary to metastatic carcinoma of the prostate; 2002. www.jfponline.com/Pages.

8. Arya B., Ghazi-Moghadam B., Goolalipour MJ, Azarhoosh R. Acute appendicitis due to metastasis of prostatic carcinoma. Medical Journal of the Islamic Republic of Iran. 2003;17(3):263-265.

9. Ozyazici S, Karateke F, Menekse E, Das K, Demirturk P, 
Ozdogan M. Metastasis from prostatic carcinoma causing acute appendicitis: Report of a case. Int J Surg Case Rep. 2013;4(4):409-411.
10. Blair NP, Bugis SP, Turner LJ, MacLeod MM. Review of the pathologic diagnoses of 2,216 appendectomy specimens. Am J Surg. 1993;165(5):618-620. 